Supplementary Information

\title{
Superhydrophobic Self-cleaning Hierarchical Micro/Nano Nanocomposite Coating with High Corrosion Resistance and Durability
}

\author{
Zhipeng Jin, Hui Mei※, Longkai Pan, Hongxia Liu, Laifei Cheng \\ Science and technology on Thermostructural Composite Materials Laboratory, School \\ of Materials Science and Engineering, Northwestern Polytechnical University, Xi'an \\ Shaanxi 710072, China \\ Corresponding author. E-mail address: phdhuimei@yahoo.com (H. Mei).
}

\section{Contents of the Supporting Information}

Total number of pages: 6

Total number of figures: 7

Total number of Video: 1 


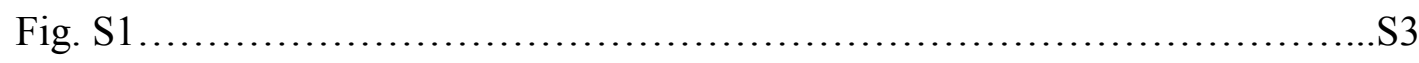

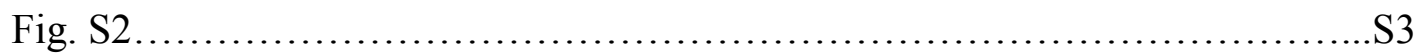

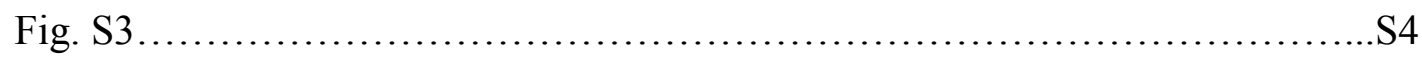

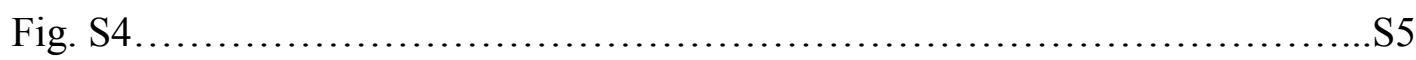

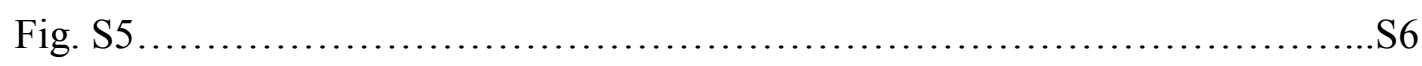

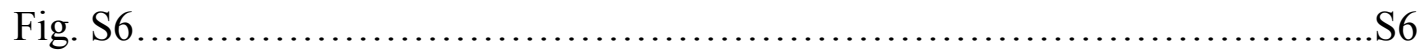

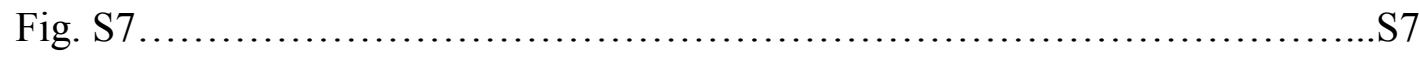

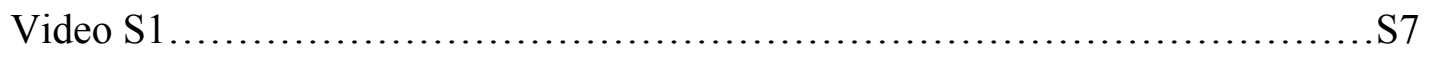



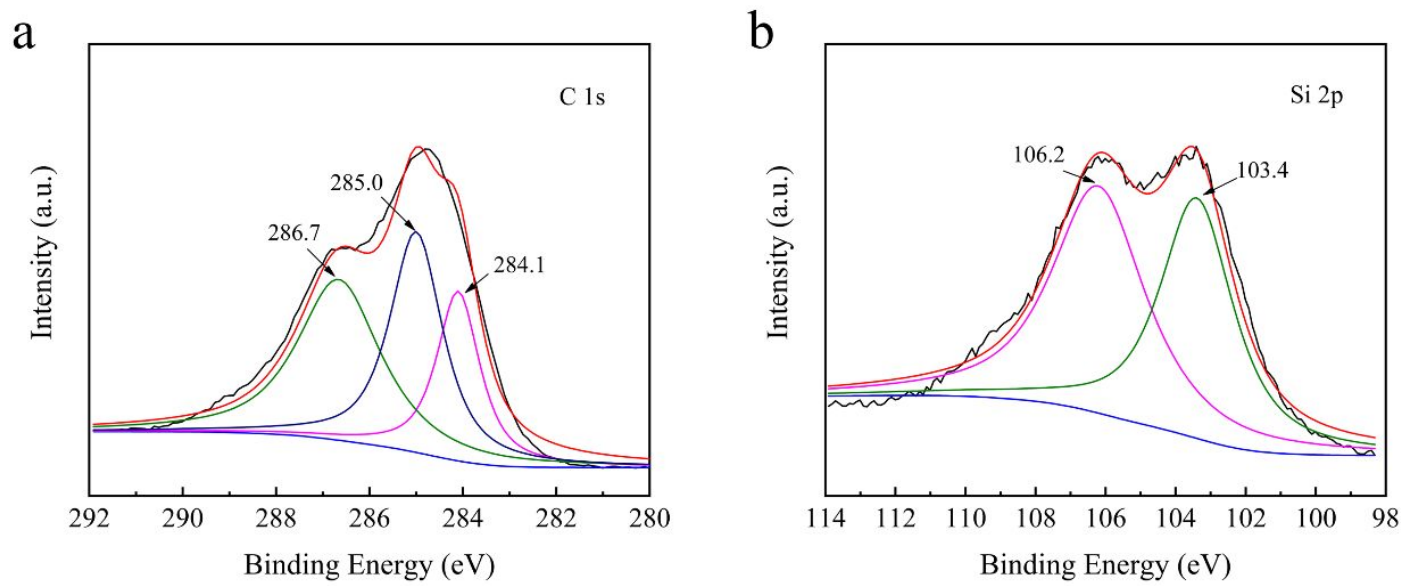

Fig. S1 XPS analysis of ZIF-8/SiO $/$ PDMS coating, (a) and (b) corresponding high resolution spectra in the $\mathrm{C} 1 \mathrm{~s}$ and $\mathrm{Si} 2 \mathrm{p}$ region.

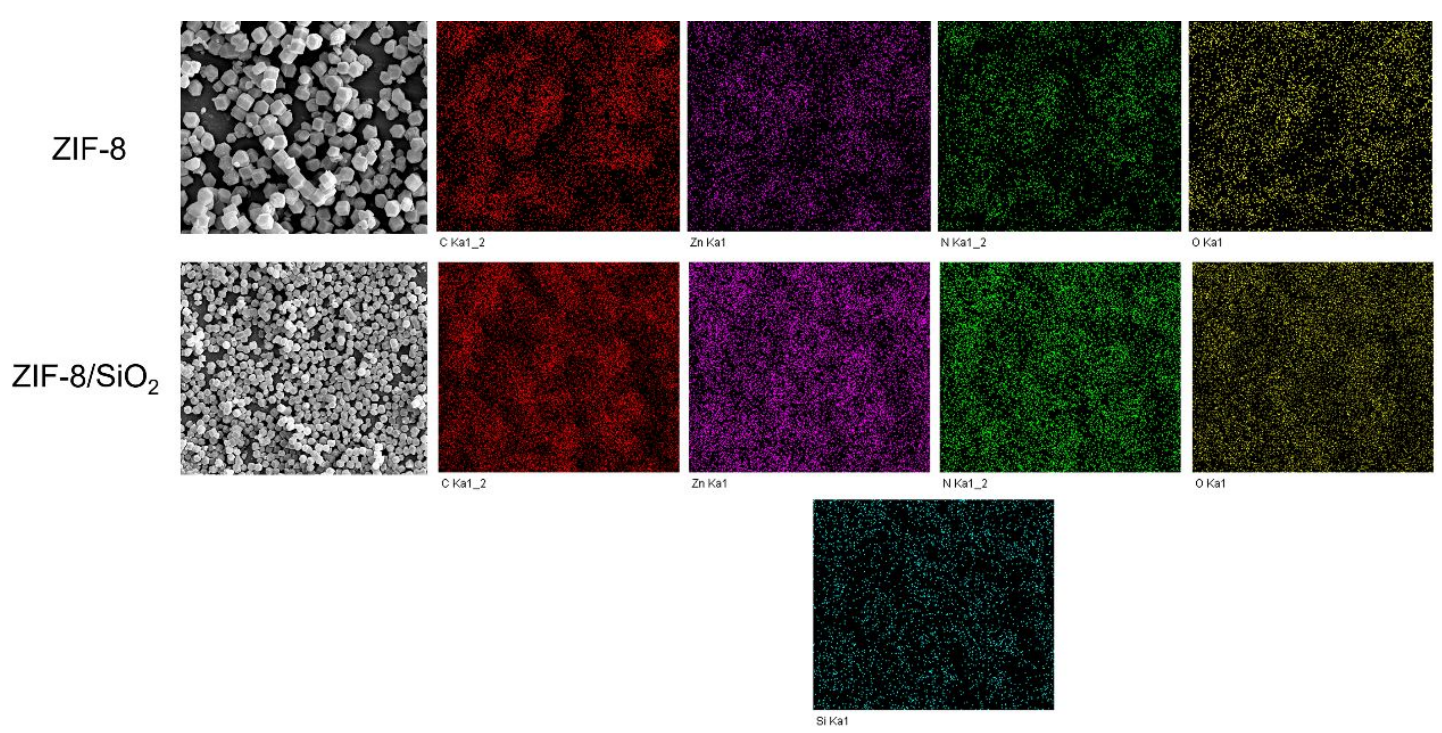

Fig. S2 The EDS spectrum of the ZIF-8 and the ZIF-8/SiO 2 particles. 


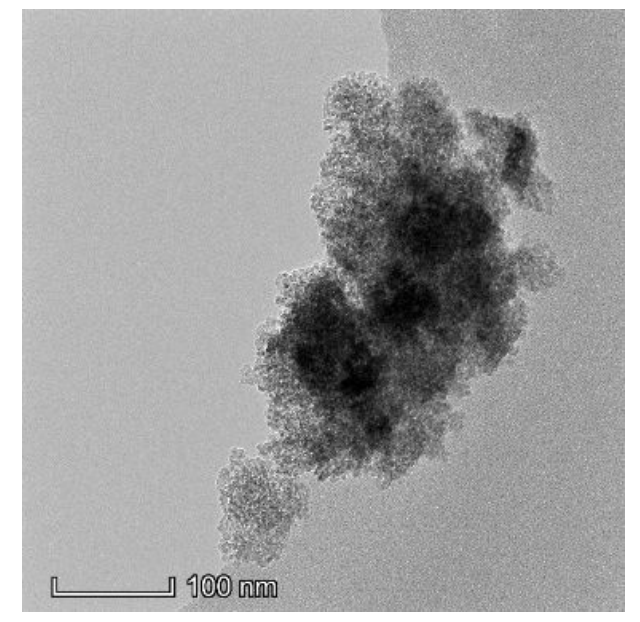

Fig. S3 TEM images of the $\mathrm{ZIF}-8 / \mathrm{SiO}_{2}$ particles. 

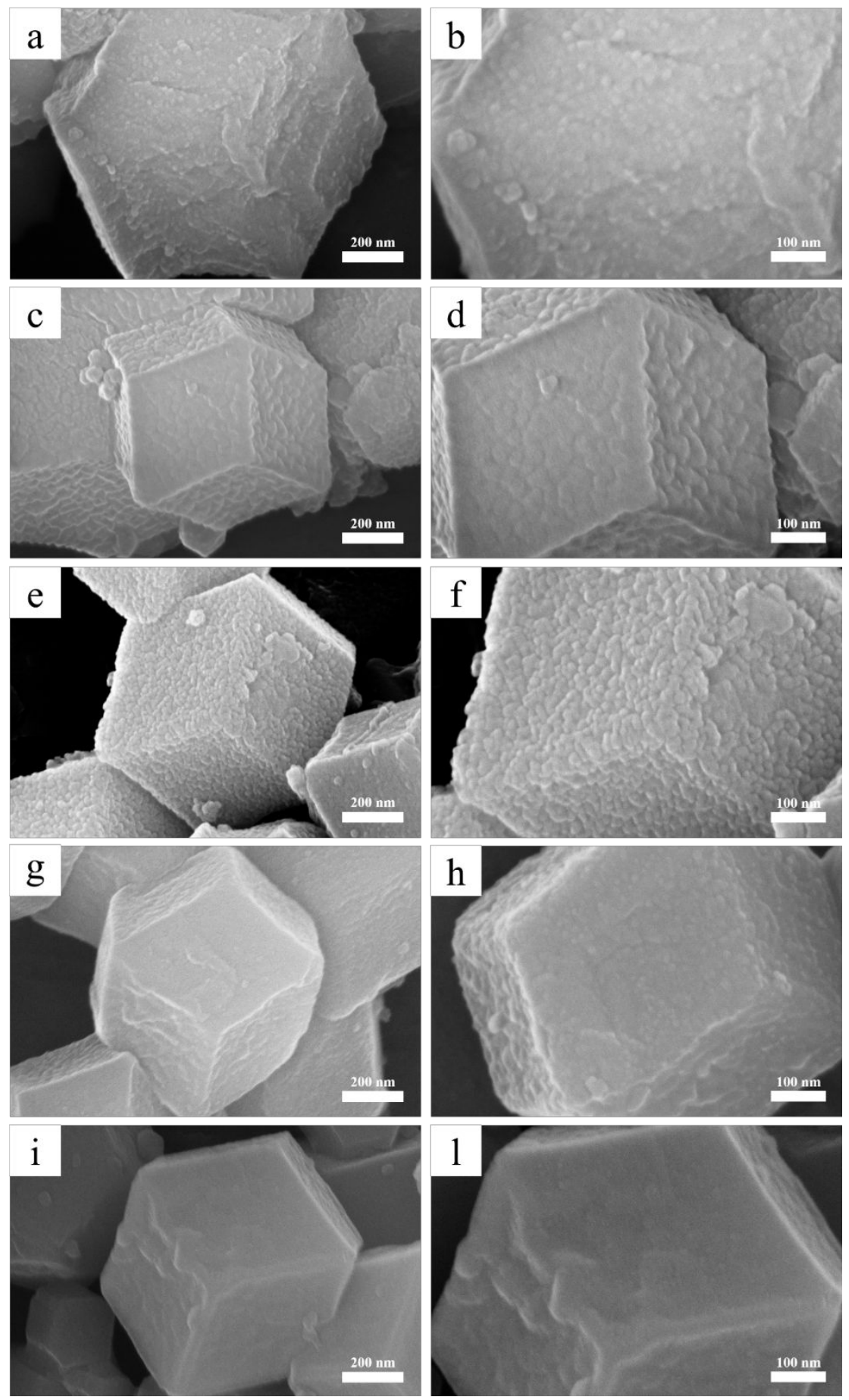

Fig. S4 SEM images of $\mathrm{ZIF}-8 / \mathrm{SiO}_{2}$ particles made using different concentrations of

TEOS and the volume ratio of TEOS are (a-b) $0.1 \%$, (c-d) $0.15 \%$, (e-f) $\mathbf{0 . 2 \%}$, (g-h)

$$
0.25 \% \text {, (i-1) } 0.3 \% \text {. }
$$



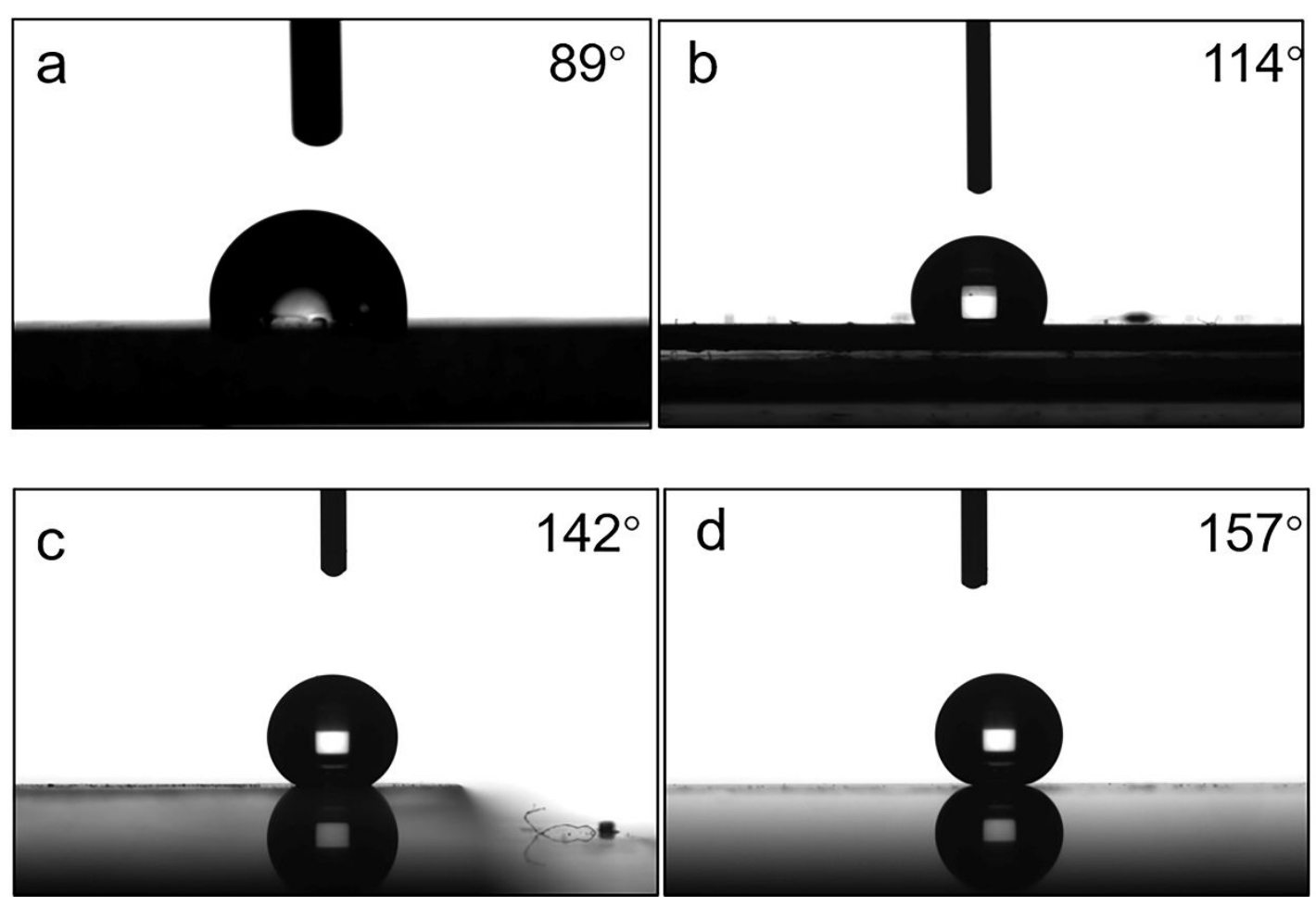

Fig. S5 The water contact angle (CA) of (a) the bare Al surface, (b) ZIF-8, (c) ZIF8/SiO 2 particles and (d) the $\mathrm{ZIF}-8 / \mathrm{SiO}_{2} / \mathrm{PDMS}$ surface.

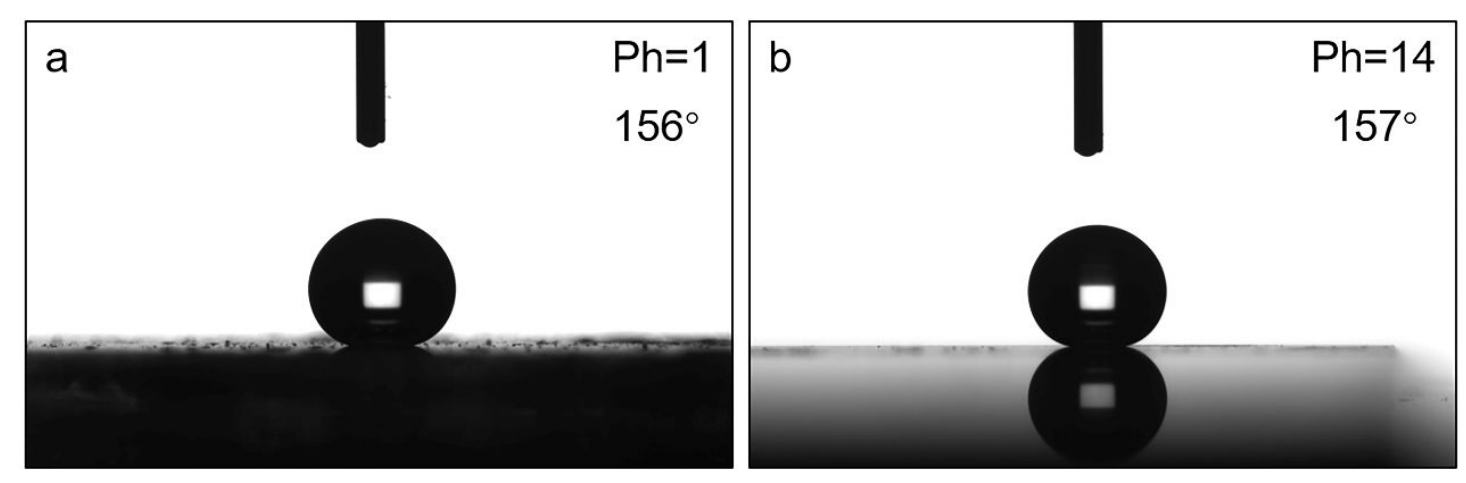

Fig. S6 The water contact angle (CA) of (a) $\mathrm{Ph}=1$, (b) $\mathrm{pH}=14$. 

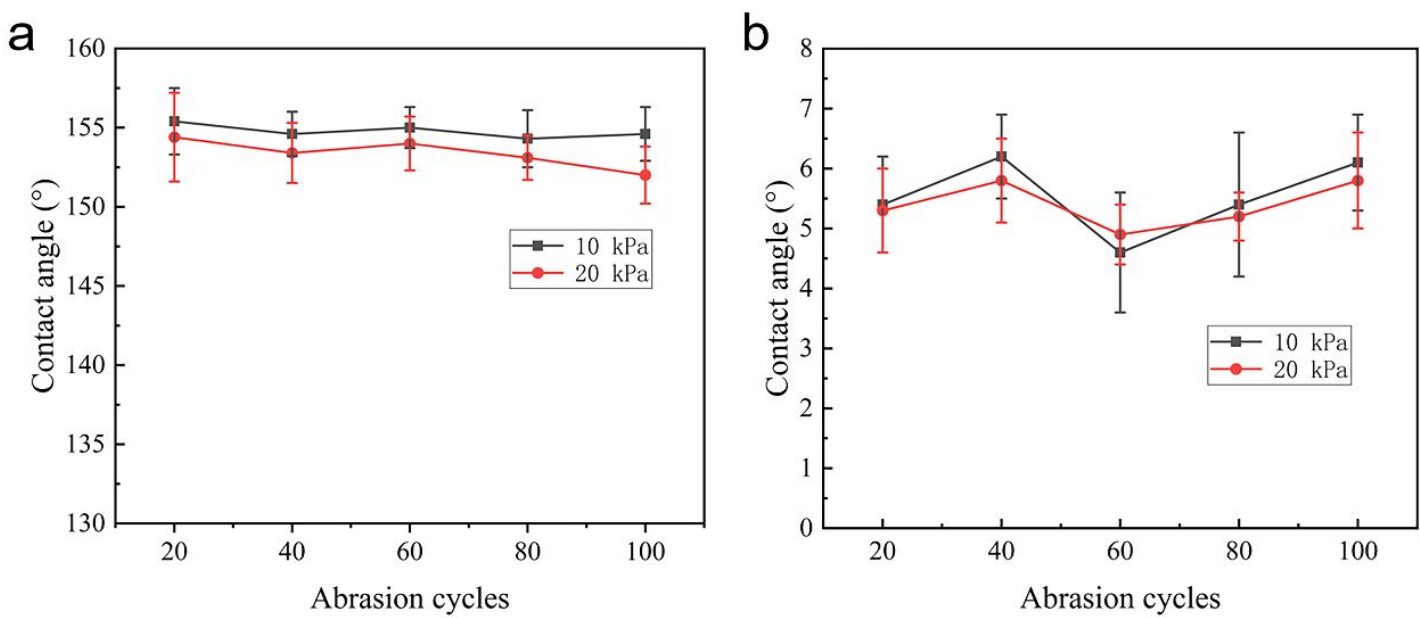

Fig. S7 The relationship between water CA (a) and SA (b) with abrasion cycle number under different pressures.

Video S1: Self-cleaning test on different samples. Al substrate (left) and the superhydrophobic surfaces (right). 